\title{
Efeitos do treinamento muscular do assoalho pélvico em mulheres com prolapso de órgãos pélvicos: uma revisão sistemática
}

\section{Effects of pelvic floor muscle training on women with pelvic organ failure: a systematic review}

\author{
Ana Carla Da Silva Pedra ${ }^{1}$ \\ Paloma De Oliveira Brasileiro ${ }^{1 z}$ \\ Taynara Do Carmo Silva ${ }^{1}$ \\ André Luiz Cordeiro Lisboa ${ }^{2}$
}

RESUMO

Introdução: 0 prolapso de órgãos pélvicos (POP) se caracteriza pelo declínio da parede vaginal, ápice da vagina ou útero. Uma disfunção decorrente do enfraquecimento dos músculos do assoalho pélvico (MAP).O treinamento muscular do assoalho pélvico (PFMT) visa melhorar a função muscular, atuando no fortalecimento do MAP e impedindo o deslocamento descendente dos órgãos. Objetivo: Revisar os efeitos sistêmicos do treinamento muscular do assoalho pélvico em mulheres com prolapso de órgãos pélvicos. Metodologia: Esta é uma revisão sistemática. Foi realizada uma busca nas bases de dados MEDLINE (PubMed), LILACS e SciELO, sem restrições relacionadas ao ano de publicação. Apenas ensaios clínicos randomizados foram incluídos neste estudo, que discorreram sobre o treinamento muscular do assoalho pélvico em mulheres com prolapso de órgãos pélvicos. Excluídos estudos que continham tratamentos em adolescentes, associado à medicaçãoou métodos cirúrgicos, que não abordavam o treinamento muscular como tratamento para o prolapso, pesquisa com mulheres que realizam apenas musculação, relatos de casos e observações clínicas. Foram utilizados os seguintes descritores: "Prolapso" AND "tratamento" AND“assoalho pélvico" AND "fortalecimento". Resultados: Dos seis artigos escolhidos para fazerem parte desta revisão sistemática, cinco apresentam resultados significativos em relação a utilização do PFMT impedem a piora do prolapso, melhoram os sintomas e qualidade de vida das mulheres. Apenas um não mostrou um possível benefício do treinamento muscular no prolapso. Conclusão: 0 treinamento muscular do assoalho pélvico (PFMT) gera efeitos positivos, proporcionando qualidade de vida para mulheres com prolapso quando realizado corretamente e com acompanhamento profissional.

\section{PALAVRAS-CHAVE}

Prolapso, tratamento, assoalho pélvico, fortalecimento.

\footnotetext{
1 Faculdade Nobre, Feira de Santana - Bahia, Brasil.

2 Escola Bahiana de Medicina e Saúde Pública, Salvador - Bahia, Brasil
} 


\section{ABSTRACT}

Introduction: Pelvic organ prolapse (POP) is characterized by the decline of the vaginal wall, apex of the vagina or uterus. A dysfunction resulting from weakening of the pelvic floor muscles (PAM). Pelvic floor muscle training (PFMT) aims to improve muscle function, strengthening MAP and preventing downward displacement of organs. Objective: To review the systemic effects of pelvic floor muscle training in women with pelvic organ prolapse. Methodology: This is a systematic review. We searched the MEDLINE (PubMed), LILACS and SciELO databases without restrictions related to the year of publication. Only randomized controlled trials were included in this study, which discussed pelvic floor muscle training in women with pelvic organ prolapse. Studies that included treatments in adolescents, associated with medication or surgical methods, that did not address muscle training as treatment for prolapse, research with women who perform only weight training, case reports and clinical observations were excluded. The following descriptors were used: "Prolapse" AND "treatment" AND "pelvic floor" AND "strengthening". Results: Of the six articles chosen to be part of this systematic review, five presented significant results regarding the use of PFMT, preventing worsening of prolapse, improving women's symptoms and quality of life. Only one did not show a possible benefit of muscle training in prolapse. Conclusion: Pelvic floor muscle training (PFMT) has positive effects, providing quality of life for women with prolapse when performed correctly and with professional monitoring.

\section{KEYWORDS}

Prolapse, treatment, pelvic floor, strengthening. 


\section{INTRODUÇÃo}

Caracterizado pelo declínio da parede vaginal anterior e posterior, do ápice da vagina ou do útero, o prolapso de órgãos pélvicos (POP) acomete cerca de 30\% a $50 \%$ das mulheres multíparas com idade superior a 50 anos (WIEGERSMA et al. 2014).Classificado em graus, o POP apresenta características que variam de acordo com sua classificação.Pode ser assintomático, ou apresentar sintomas como: pressão ou peso pélvico, dor pélvica e problemas urinários ou intestinais, sangramento, corrimento e disfunção anorretal (FERREIRA et al. 2018 e CHANG et al. 2019).

POP é uma das disfunções decorrentedo enfraquecimento dos músculos do assoalho pélvico (MAP). Esses músculos desempenham um papel fundamental na sustentação dos órgãos pélvicos, consequentemente o enfraquecimento do MAP está vigorosamente associado à idade avançada e partos recorrentes. Deve-se também considerar fatores como: história familiar, obesidade, trabalhos que requerem muito esforço e maus hábitos (FLUSBERG et al. 2019 e HAGEN et al. 2014).

0 prolapso acarreta disfunção sexual, afeta as atividades de vida diária, gera constrangimento no ato sexual, afetando a qualidade de vida das mulheres. Como forma de intervenção para o POP, pode-se considerar para tratamento o método conservador, que se refere ao uso de pessário vaginal (um instrumento utilizado para apoiar os órgãos do assoalho pélvico) e o treinamento da musculatura do assoalho pélvico (PFMT), sendo mais eficaz no estágio leve a moderado. Em seu grau mais avançado o método cirúrgico é o recomendado (WIEGERSMAA et al. 2014).

O PFMT é eficaz na prevenção, tratamento do prolapso e pós-operatório. Este visa melhorar a função muscular, atuando no fortalecimento de toda musculatura e impedindo o deslocamento descendente dos órgãos. 0 treinamento realizado em longo prazo permite que o assoalho pélvico volte a ter suporte estrutural adequado para sustentá-los (PANMAN et al. 2017).

É de suma importância que as mulheres sejam orientadas por um fisioterapeuta especialista em saúde da mulher quanto à eficácia do PFMT tanto na prevenção como no tratamento do POP e das demais disfunções que acometem o MAP. Os efeitos do treinamento muscular do assoalho pélvico visam o aumento do tônus muscular e a consciência corporal na contração voluntária antes e durante um aumento da pressão intra-abdominal a fim de impedir a descida de órgãos pélvicos (MCCLURG et al. 2014).
Dado o contexto, a forma de tratamento mais eficaz e de baixo custo econômico é o PFMT, pois há estudos e evidências comprovadas de melhora e manutenção da musculatura do assoalho, diante desta patologia. Sendo recomendado como intervenção principal devidoefeito significativo na prevenção do POP (MAXWELL et al. 2017 e BRANDT et al. 2019).

0 objetivo desta pesquisa foi revisarsistematicamente os efeitos do treinamento muscular do assoalho pélvico em mulheres com prolapso de órgãos pélvicos.

\section{METODOLOGIA}

\section{Estratégia de busca para identificação dos estudos}

Trata-se de uma revisão sistemática de literatura, que foi realizada através de busca nas bases de dados MEDLINE (PubMed), LILACS e SciELO. Os artigos selecionados foram nos idiomas: inglês e português, sem restrição em relação ao ano de publicação. Foram utilizados os operadores booleanos and e or. As palavras chave utilizadas foram: Prolapso "Prolapse", tratamento "treatment", assoalho pélvico "pelvicfloor", fortalecimento "strengthening". Sendo definidas com base nos descritores em Ciências da Saúde (Decs) e seus correspondentes na língua inglesa no Medical SubjectHeadings (MeSH).

\section{Critérios de elegibilidade}

Para critérios de inclusão foram consultados ensaios clínicos que discorreram sobre o treinamento muscular do assoalho pélvico em mulheres com prolapso de órgãos pélvicos. Foram excluídos os estudos que continham tratamentos em adolescentes, associadoà medicação ou a métodos cirúrgicos, estudosque não abordavam o treinamento muscular do assoalho pélvico como tratamento para o prolapso,pesquisa com mulheres que realizam apenas musculação, relatos de casos e observações clínicas.

\section{Avaliação da qualidade metodológica}

Foi examinada a qualidade metodológica do trabalho, através da base de dados de Revisões Sistemáticas Cochrane (CDSR), uma biblioteca que possui diferentes tipos de evidências, livre e de boa qualidade, tornando todo indicativo acessível, criado pelo manual da Cochrane para revisões sistemáticas de intervenções, versão 5.1.0. Foram usados os sete domínios para avaliação: tipos de randomização; sigilo de alocação; cegamento; análise por intenção de tratar; parada precoce por be- 
nefício; descrição seletiva do desfecho e escala validada. Sendo que os riscos de viés foram classificados em "baixo risco de viés", "alto risco de viés" e "incerto risco de viés" (CARVALHO et al. 2013).

\section{Extração dos dados}

A princípio, a seleção de estudos para extrair os dados necessários foi baseada na verificação de títulos dos artigos, assim como na análise dos resumos disponíveis dos mesmos. Posteriormente, os relatórios completos do estudo foram comparados com os parâmetros de inclusão já estabelecidos para determinar sua significância para a revisão sistemática. Dois revisores, de forma autônoma e cega, atendendo aos critérios de inclusão e exclusão previamente definidos no protocolo de pesquisa, foram extraídos os dados para exami- nar as propriedades do estudo, os pacientes e a possível eficácia dos tratamentos.

\section{RESULTADOS}

Através dos critérios de busca de artigos sobre o tema foram encontrados 24 estudos. Após a leitura desses estudos e a partir dos critérios de exclusão, 18 desses foram excluídos por não abordarem o que estava proposto para a revisão (04 não abordavam o treinamento muscular do assoalho pélvico como tratamento principal, 09 estavam associados a medicamentos oua métodos cirúrgicos, 05 envolviammulheres que realizaram apenas musculação), resultando em 06 artigos com os devidos critérios de inclusão pra análise final.A Figura 1 mostra o processo de seleção dos artigos através dofluxograma da plataforma PRISMA.

Figura 1: Fluxograma para obter os resultados. por outras fontes $(n=03)$

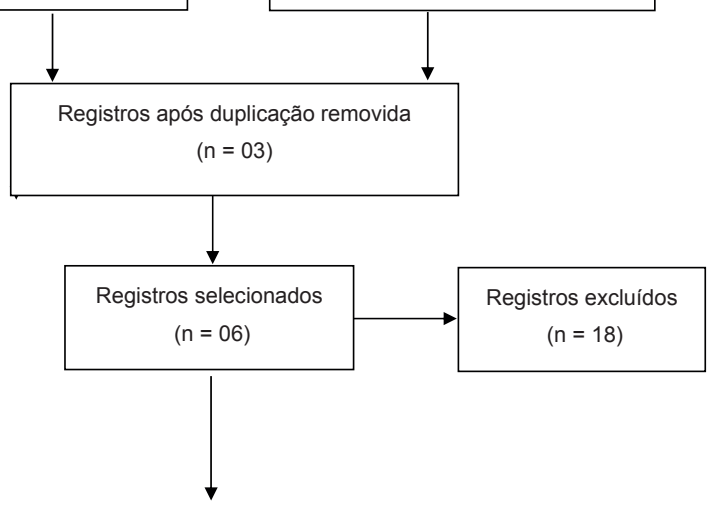

\begin{tabular}{|c|c|}
\hline $\begin{array}{l}\text { Artigos de texto completo } \\
\text { avaliados para } \\
\text { elegibilidade } \\
(n=06)\end{array}$ & $\begin{array}{l}\text { Artigos de texto completo } \\
\text { excluídos, com motivos: } \\
\qquad(n=18) \\
\text { - Não abordavam o }\end{array}$ \\
\hline$\nabla$ & $\begin{array}{l}\text { assoalho pélvico como } \\
\text { tratamento principal } \\
(\mathrm{n}=04)\end{array}$ \\
\hline $\begin{array}{l}\text { Estudos incluídos na } \\
\text { síntese qualitativa } \\
\qquad(n=05)\end{array}$ & $\begin{array}{l}\text { - Estavam associados a } \\
\text { medicamentos ou a } \\
\text { métodos cirúrgicos } \\
\text { (n=09) }\end{array}$ \\
\hline & $\begin{array}{l}\text { - Envolviam mulheres que } \\
\text { realizaram apenas } \\
\text { musculação } \\
(n=05)\end{array}$ \\
\hline
\end{tabular}


A ferramenta Cochrane Collaboration foi utilizada para avaliar a qualidade metodológica dos seis artigos selecionados (Quadro 1). Os mesmos foram avaliados com os critérios: alto risco de viés, baixo risco de viés e risco incerto de viés. Os seis estudos apresentadosnesta revisão sistemática discorrem sobre os efeitos do treinamento muscular do assoalho pélvico, em mulheres com prolapso de órgãos pélvico.

Quadro 1: Análise metodológica da qualidade dos estudos: risco de viés de cada estudo com base na ferramenta Cochrane Collaboration.

\begin{tabular}{|c|c|c|c|c|c|c|}
\hline & $\begin{array}{l}\text { Hagen et } \\
\text { al. (2014) }\end{array}$ & $\begin{array}{l}\text { Panmanet al. } \\
\text { (2017) }\end{array}$ & $\begin{array}{l}\text { Bo Kari et } \\
\text { al. (2015) }\end{array}$ & $\begin{array}{l}\text { Jarvis et al. } \\
\text { (2005) }\end{array}$ & $\begin{array}{l}\text { Bernardes et } \\
\text { al. (2012) }\end{array}$ & $\begin{array}{l}\text { Piya-Anant } \\
\text { et al. } \\
(2003)\end{array}$ \\
\hline $\begin{array}{l}\text { Tipo de } \\
\text { randomização }\end{array}$ & $\begin{array}{l}\text { Baixo risco } \\
\text { de viés }\end{array}$ & $\begin{array}{l}\text { Baixo risco } \\
\text { de viés }\end{array}$ & $\begin{array}{l}\text { Risco in- } \\
\text { certo de } \\
\text { viés }\end{array}$ & $\begin{array}{l}\text { Baixo risco } \\
\text { de viés }\end{array}$ & $\begin{array}{l}\text { Baixo risco de } \\
\text { viés }\end{array}$ & $\begin{array}{l}\text { Baixo risco } \\
\text { de viés }\end{array}$ \\
\hline $\begin{array}{l}\text { Segredo de aloca- } \\
\text { ções }\end{array}$ & $\begin{array}{l}\text { Baixo risco } \\
\text { de viés }\end{array}$ & $\begin{array}{l}\text { Baixo risco } \\
\text { de viés }\end{array}$ & $\begin{array}{l}\text { Baixo ris- } \\
\text { co de viés }\end{array}$ & $\begin{array}{l}\text { Baixo risco } \\
\text { de viés }\end{array}$ & $\begin{array}{l}\text { Baixo risco de } \\
\text { viés }\end{array}$ & $\begin{array}{l}\text { Baixo risco } \\
\text { de viés }\end{array}$ \\
\hline Cegamento & $\begin{array}{l}\text { Risco in- } \\
\text { certo de } \\
\text { viés }\end{array}$ & $\begin{array}{l}\text { Risco incer- } \\
\text { to de viés }\end{array}$ & $\begin{array}{l}\text { Alto risco } \\
\text { de viés }\end{array}$ & $\begin{array}{l}\text { Risco incer- } \\
\text { to de viés }\end{array}$ & $\begin{array}{l}\text { Alto risco de } \\
\text { viés }\end{array}$ & $\begin{array}{l}\text { Risco incer- } \\
\text { to de viés }\end{array}$ \\
\hline $\begin{array}{l}\text { Intenção de tratar a } \\
\text { análise }\end{array}$ & $\begin{array}{l}\text { Baixo risco } \\
\text { de viés }\end{array}$ & $\begin{array}{l}\text { Baixo risco } \\
\text { de viés }\end{array}$ & $\begin{array}{l}\text { Baixo ris- } \\
\text { co de viés }\end{array}$ & $\begin{array}{l}\text { Baixo risco } \\
\text { de viés }\end{array}$ & $\begin{array}{l}\text { Baixo risco de } \\
\text { viés }\end{array}$ & $\begin{array}{l}\text { Baixo risco } \\
\text { de viés }\end{array}$ \\
\hline $\begin{array}{l}\text { Parada antecipada } \\
\text { para benefício }\end{array}$ & $\begin{array}{l}\text { Alto risco } \\
\text { de viés }\end{array}$ & $\begin{array}{l}\text { Alto risco de } \\
\text { viés }\end{array}$ & $\begin{array}{l}\text { Alto risco } \\
\text { de viés }\end{array}$ & $\begin{array}{l}\text { Alto risco } \\
\text { de viés }\end{array}$ & $\begin{array}{l}\text { Alto risco de } \\
\text { viés }\end{array}$ & $\begin{array}{l}\text { Alto risco } \\
\text { de viés }\end{array}$ \\
\hline $\begin{array}{l}\text { Descrição seletiva } \\
\text { do resultado }\end{array}$ & $\begin{array}{l}\text { Baixo risco } \\
\text { de viés }\end{array}$ & $\begin{array}{l}\text { Baixo risco } \\
\text { de viés }\end{array}$ & $\begin{array}{l}\text { Risco in- } \\
\text { certo de } \\
\text { viés }\end{array}$ & $\begin{array}{l}\text { Baixo risco } \\
\text { de viés }\end{array}$ & $\begin{array}{l}\text { Risco incerto } \\
\text { de viés }\end{array}$ & $\begin{array}{l}\text { Baixo risco } \\
\text { de viés }\end{array}$ \\
\hline Escala validada & $\begin{array}{l}\text { Risco in- } \\
\text { certo de } \\
\text { viés }\end{array}$ & $\begin{array}{l}\text { Risco incer- } \\
\text { to de viés }\end{array}$ & $\begin{array}{l}\text { Risco } \\
\text { incerto de } \\
\text { viés }\end{array}$ & $\begin{array}{l}\text { Baixo risco } \\
\text { de viés }\end{array}$ & $\begin{array}{l}\text { Baixo risco de } \\
\text { viés }\end{array}$ & $\begin{array}{l}\text { Risco incer- } \\
\text { to de viés }\end{array}$ \\
\hline
\end{tabular}

Dos seis artigos que escolhidos para fazerem parte desta revisão sistemática, cinco deles apresentam resultados significativos em relação a utilização de treinamento da musculatura do assoalho pélvico na melhora dos sintomas e impedem a piora do prolapso de órgãos pélvicos, bem como na qualidade de vida de mulheres com esta patologia. Apenas um dos estudos relata que não há melhora dos sintomas de POP e não mostrou um possível benefício da utilização do treinamento como melhora do prolapso de órgãos pélvicos. 0 Quadro 2 mostra os principais ensaios clínicos sobre o assunto e seus resultados. 
Quadro 2. Dados gerais sobre os artigos selecionados para obtenção dos resultados.

\begin{tabular}{|c|c|c|c|c|}
\hline Autor & Objetivo & Amostra & Intervenção & Conclusão \\
\hline $\begin{array}{c}\text { Hagenet } \\
\text { al.(2014) }\end{array}$ & $\begin{array}{l}\text { Estabelecer a eficácia do } \\
\text { treinamento individualizado } \\
\text { dos músculos do assoalho } \\
\text { pélvico individual para } \\
\text { reduzir os sintomas do } \\
\text { prolapso. }\end{array}$ & $\begin{array}{l}\text { GI: } 225 \\
\text { GC: } 222\end{array}$ & $\begin{array}{l}\text { GI: Receberam um } \\
\text { programa individualizado } \\
\text { de treinamento muscular } \\
\text { do assoalho pélvico ou um } \\
\text { folheto de conselhos sobre } \\
\text { estilo de vida. } \\
\text { GC: Nenhum treinamento } \\
\text { muscular. }\end{array}$ & $\begin{array}{l}\text { O treinamento muscular } \\
\text { individual do prolapso } \\
\text { do assoalho pélvico é } \\
\text { eficaz para melhorar os } \\
\text { sintomas do prolapso. }\end{array}$ \\
\hline $\begin{array}{c}\text { Panman et } \\
\text { al.(2017) }\end{array}$ & $\begin{array}{l}\text { Comparar efeitos e custo } \\
\text { efetividade do treinamento } \\
\text { muscular do assoalho } \\
\text { pélvico (PFMT) e espera } \\
\text { vigilante. }\end{array}$ & $\begin{array}{l}\text { GI: } 145 \\
\text { GC: } 142\end{array}$ & $\begin{array}{l}\text { GI: Treinamento muscular } \\
\text { do assoalho pélvico. } \\
\text { GC: Espera vigilante. }\end{array}$ & $\begin{array}{l}\text { O PFMT resultou em } \\
\text { maior melhora dos } \\
\text { sintomas do assoalho } \\
\text { pélvico em comparação } \\
\text { com a espera vigilante. }\end{array}$ \\
\hline $\begin{array}{c}\text { Bo Kari et al. } \\
\text { (2015) }\end{array}$ & $\begin{array}{l}\text { Avaliar o efeito do } \\
\text { treinamento muscular do } \\
\text { assoalho pélvico na } \\
\text { prevenção e tratamento de } \\
\text { sintomas e sinais de POP. }\end{array}$ & $\begin{array}{l}\text { GI: } 87 \\
\text { GC: } 88\end{array}$ & $\begin{array}{l}\text { GI: Recomendadas a } \\
\text { realizar o treinamento por } \\
4 \text { meses liderado por } \\
\text { fisioterapeutas. } \\
\text { GC: Não teve } \\
\text { acompanhamento durante } \\
\text { o período. }\end{array}$ & $\begin{array}{l}\text { O treinamento não } \\
\text { melhorou os sintomas } \\
\text { de POP, suporte da } \\
\text { bexiga ou protuberância } \\
\text { vaginal nesta população } \\
\text { de mulheres primíparas } \\
\text { após o parto vaginal. }\end{array}$ \\
\hline $\begin{array}{l}\text { Jarvis et } \\
\text { al.(2005) }\end{array}$ & $\begin{array}{l}\text { Investigar o papel da } \\
\text { fisioterapia perioperatória } \\
\text { em mulheres submetidas a } \\
\text { cirurgia corretiva para } \\
\text { prolapso de órgãos pélvicos. }\end{array}$ & $\begin{array}{l}\text { GI: } 30 \\
\text { GC: } 30\end{array}$ & $\begin{array}{l}\text { GI: foram submetidas a } \\
\text { fisioterapia pré-operatória. } \\
\text { GC: não tiveram } \\
\text { fisioterapia antes de sua } \\
\text { cirurgia de prolapso. }\end{array}$ & $\begin{array}{l}\text { As intervenções } \\
\text { rotineiras de } \\
\text { fisioterapia pré e pós- } \\
\text { operatórias melhoram } \\
\text { os resultados físicos e a } \\
\text { qualidade de vida em } \\
\text { mulheres submetidas a } \\
\text { cirurgia corretiva de } \\
\text { prolapso de órgãos } \\
\text { pélvicos. }\end{array}$ \\
\hline $\begin{array}{l}\text { Bernardes et } \\
\text { al.(2012) }\end{array}$ & $\begin{array}{l}\text { Avaliar a eficácia do } \\
\text { treinamento muscular do } \\
\text { assoalho pélvico e exercícios } \\
\text { hipopressivos para aumentar } \\
\text { a CSA do músculo elevador } \\
\text { do ânus. }\end{array}$ & $\begin{array}{l}\text { GI1: } 21 \\
\text { GI2: } 21 \\
\text { GC: } 16\end{array}$ & $\begin{array}{l}\text { GI1: Treinamento do MAP. } \\
\text { GI2: Exercícios } \\
\text { hipopressivos mais } \\
\text { contração voluntária de } \\
\text { MAP. } \\
\text { GC: Contração dos } \\
\text { músculos do assoalho } \\
\text { pélvico sem protocolo } \\
\text { definido. }\end{array}$ & $\begin{array}{l}\text { O treinamento dos } \\
\text { músculos do assoalho } \\
\text { pélvico e exercícios } \\
\text { hipopressivos parecem } \\
\text { produzir melhorias } \\
\text { semelhantes na CSA do } \\
\text { músculo elevador do } \\
\text { ânus. }\end{array}$ \\
\hline $\begin{array}{l}\text { Piya-Anant et } \\
\text { al.(2003) }\end{array}$ & $\begin{array}{l}\text { Determinar a eficácia do } \\
\text { exercício do assoalho pélvico } \\
\text { para evitar a piora do } \\
\text { prolapso genital em } \\
\text { mulheres idosas. }\end{array}$ & $\begin{array}{l}\text { GI: } 330 \\
\text { GC: } 324\end{array}$ & $\begin{array}{l}\text { GI: recebeu treinamento no } \\
\text { exercício do assoalho } \\
\text { pélvico e foi solicitado a } \\
\text { realizar o exercício } 30 \\
\text { vezes após uma refeição, } \\
\text { todos os dias durante } 24 \\
\text { meses. } \\
\text { GC: observado pelo } \\
\text { período de } 24 \text { meses. }\end{array}$ & $\begin{array}{l}\text { Um programa de } \\
\text { exercícios do assoalho } \\
\text { pélvico de } 24 \text { meses foi } \\
\text { eficaz para evitar a } \\
\text { piora do prolapso } \\
\text { genital nas mulheres } \\
\text { que tiveram prolapso } \\
\text { genital grave. }\end{array}$ \\
\hline
\end{tabular}

GI: Grupo Intervenção; GC: Grupo Controle; POP: Prolapso de órgão pélvico; MAP: Músculos do assoalho pélvico; PFMT: Treinamento muscular do assoalho pélvico; CSA: Área transvesal. 


\section{DISCUSSÃO}

Esta revisão sistemática visou demonstrar os efeitos do treinamento muscular do assoalho pélvico (PFMT) em mulheres com prolapso de órgãos pélvicos, sendo abordado como método preventivo, ou evitando a piora dos sintomas e do quadro clínico ou no tratamento. E, observou-se nos estudos selecionados que o PFMT quando comparado com mulheres que não realizam nenhum tipo de intervenção, traz melhoria aos sintomas apresentadosno POP como: pressão ou peso pélvico, dor pélvica e problemas urinários ou intestinais, sangramento, corrimento e disfunção anorretal.

0 treinamento muscular do assoalho pélvico tem benefícios namelhorados sintomas e são eficazes na reduçãoda piora no quadro de POP, no entanto os resultados variam de acordo com o tipo de prolapso, sua gravidade, quantidade de gestações e período pós-parto até o momento da abordagem.

Segundo Hagen et al.(2014)o objetivo do treinamento muscular é melhorar a função dos músculos do assoalho pélvico, trabalhando a força, resistência e coordenação e, finalmente aumentar o suporte estrutural para os órgãos pélvicos.E, da mesma maneira,Panman et al. (2017), Jarvis et al. (2005), Bernardes et al. (2012) ePiya-Anant et al. (2003)ratificam em seus estudos que o fortalecimento da musculatura do assoalho pélvico tem resultados positivosrelacionados a melhora dos sintomas do prolapso quando realizado o PFMT como tratamento. Visto que o fortalecimento proporciona resistência da cadeia muscular garantindo sustentação adequada paramanter os órgãos apoiados e diminuindo o sintoma principal que é a descida da parede vaginal.

Diante dos resultados relacionados ao benefício do PFMT em mulheres com POP, Panman et al. (2017)comentam que o treinamento conduziu com mais frequência à melhora dos sintomas, custos mais baixos das compressas absorventes e foi mais eficaz em mulheres que experimentavam maior sofrimento, sendo aconselhado em casos de incômodos do prolapso leve.Jarvis et al. (2005) relatam que com intervenções rotineiras de fisioterapia pré e pós-operatórias melhoram os resultados físicos relacionado a compressão muscular máxima do assoalho pélvico e a qualidade de vida em mulheres submetidas a cirurgia corretiva de prolapso de órgãos pélvicos.
Bernardes et al. (2012)ressalta em seu estudo um aumento significativo no volume muscular, diminuição da área de hiato genital e posição elevada da bexiga e do reto com prolapso reduzido. Apresentando melhora quando realizado o tratamento com exercícios do PFMT sob orientação de um fisioterapeuta com treinamento específicoePiya-Anantet al. (2003) comprovam que um programa de exercícios do assoalho pélvico foi eficaz para evitar a piora do quadro nas mulheres que tiveram prolapso grave, sendo justificado pelo aumento da força e resistência do MAP. Com isso percebemos a importância da realização do PFMT em mulheres com POP, pois além de atuar na redução dos sintomas, evita a piora do quadro, proporcionando qualidade de vida.

Embora a maioria dos estudos referenciasse resultados eficazes em relação ao PFMT, Bo Kari et al. (2015) abordarama prevenção e o tratamento de prolapso em mulheres puérperas primíparase não foi encontrado efeito significativo do treinamento muscular pós-parto no POP, uma vez que essa população em particular passa por processo de retorno anatômico da musculatura do assoalho pélvico ao normal após o trabalho de parto.Diante desta condição seria necessário um estudo com um tempo maior nesta população para que resultados relevantes fossem pontuados e as mulheres fossem continuamente beneficiadas com o treinamento da musculatura.

Panman et al. (2017)sugerem em seu estudo que as mulheres por falta de conhecimento sobre a patologiae receio em se expor não procuram atendimento no início dos sintomas,acarretandoredução na eficácia do treinamento muscular. Salientamos aimportância de maiores esclarecimentos sobre o prolapso de órgãos pélvicos e que as mulheres precisam ser orientadas quantoà necessidade do PFMTcomo prevenção e tratamento do POP, tendo comobenefício o fortalecimento do MAP e melhoria na qualidade de vida.

É válido ressaltar que mais estudos devem ser realizados para o melhor conhecimento sobre o PFMT como tratamento para prolapso de órgãos pélvicos, bem como a atuação do fisioterapeuta no acompanhamento das mulheres com este acometimento. Sugerimos ainda que a população poderia ser estudada a médio e longo prazo paracomprovar a eficácia do PFMT no quadro de prolapso. 


\section{CONCLUSÃO}

O treinamento muscular do assoalho pélvico gera efeitos positivosno fortalecimento e resistênciada musculatura do assoalho pélvico (MAP) favorecendo a sustentação dos órgãos pélvicos, possibilitando redução dos sintomas urinários ou intestinais, sangramento, corrimentos e alteração anorretal, pressão ou peso pélvicodecorrentes doprolapso de órgãos pélvico evitando a piora do quadro, proporcionando qualidade de vida para mulheres com prolapso de órgãos pélvicos (POP) quando realizado corretamente e com acompanhamento profissional. 


\section{REFERÊNCIAS}

Bernardes BT, Resende AP, Stupp L, Oliveira E, Castro RA, Bella $\mathrm{Z}$ et. al. Eficácia do treinamento muscular do assoalho pélvico e exercícios hipopressivos no tratamento do prolapso de órgãos pélvicos em mulheres: estudo controlado randomizado. São Paulo Med. J. 2012;130:5-9.

Bo K, Hilde G, Jensen JS, Siafarikas F, Tennfjord MK, Engh ME. Postpartum pelvic floor muscle training and pelvic organ prolapse - a randomized trial of primiparous women. American Journal of Obstetrics e Gynecology. 2015;212(38):1-7.

Brandt C,Janse van Vuuren EC. Dysfunction, activity limitations, participation restriction and contextual factors in South African women with pelvic organ prolapse. S Afr J Physiother: 2019;75(1):933.

Carvalho APV, Silva V, Grande AJ. Avaliação do risco de viés de ensaios clínicos randomizados pela ferramenta da colaboração Cochrane. Diagn Tratamento. 2013;18(1):38-44.

Chang TC, Hsiao SM, Wu PC, Chen CH, Wu WY, Lin HH. Comparison of clinical outcomes between tailored transvaginal mesh surgery and native tissue repair for pelvic organ prolapse. J Formos Med Assoc. Taiwan, 2019;118(12):1623-1632.

Ferreira HLOC, Bezerra KC, Freitas VCA, Silva TM, Moura ERF, Vasconcelos CTM et. al. Protocolo para tratamento de prolapso de órgãos pélvicos com pessário vaginal. Acta Paul Enferm. CE, 2018;31(6):585-92.

Flusberg M, Kobi M, Bahrami S,Glanc P, Palmer S, Chernyak V et. al. Multimodality imaging of pelvic floor anatomy. AbdomRadiol. NY. 2019;25.

Hagen S, Stark D, Glazener C, Dickson S, Barry S, Elders A et. al. Individualised pelvic floor muscle training in women with pelvic organ prolapse (POPPY): a multicentre randomized controlled trial. Glasgow, UK. 2014;383(9919):796-806
Jarvis SK, Hallam TK, Lujic S, Abbott JA, Vancaillie TG.Peri-operative physiotherapy improves outcomes for women undergoing incontinence and or prolapse surgery: results of a randomised controlled trial. AustN Z J ObstetGynaecol. 2005;45(4):300-3.

Maxwell M, Semple K,Wane S, Elders A, Duncan E, Abhyankar P et. al. PROPEL: implementation of an evidence based pelvic floor muscle training intervention for women with pelvic organ prolapse: a realist evaluation and outcomes study protocol. BMC Health Serv Res. 2017; 17:843.

McClurg D, Hilton P, Dolan L, Monga A, Hagen S, Frawley H et. al. Pelvic floor muscle training as an adjunct to prolapse surgery: a randomised feasibility study. Int Urogynecol J. 2014;25(7):883-91.

Panman C, Wiegersma M, Kollen BJ, Berger MY, Lisman-Van Leeuwen Y, Vermeulen KM, Dekker JH et. al. Two-year effects and cost-effectiveness of pelvicfloor muscle training in mild pelvic organprolapse: a randomised controlled trial inprimary care. BJOG. 2017;124(3):511-520.

Piya-Anant M, Therasakvichya S, Leelaphatanadit C, Techatrisak K.Integrated health research program for the Thai elderly: prevalence of genital prolapse and effectiveness of pelvic floor exercise to prevent worsening of genital prolapse in elderly women.J Med Assoc Thai. 2003;86(6):509-15.

Wiegersmaa M, Chantal MCRP, Kollena BJ, Vermeulenb KM, Schramc AJ, Messelinkd EJ et. al. Pelvic floor muscle training versus watchful waiting or pessary treatment for pelvic organ prolapse (POPPS): Design and participant baseline characteristics of two parallel pragmatic randomized controlled trials in primary care. ElsevierIreland, Maturitas 2014;77(2):168-73.

Wiegersma M, Panman CM, Kollen BJ, Berger MY, Lisman-Van Leeuwen, Dekker JH. Effect of pelvic floor muscle training compared with watchful waiting in older women with symptomatic mild pelvic organ prolapse: randomised controlled trial in primary care. BMJ. 2014;22(349):73-78. 